\title{
El Tribunal Africano de Derechos Humanos y de los Pueblos: ¿hacia un África en paz?
}

\author{
The African Court on Human and Peoples: \\ Towards an Africa in Peace?
}

\section{Juan Bautista Cartes Rodríguez*}

\begin{abstract}
SUMARIO: I. Introducción. II. Génesis del Tribunal Africano. III. Composición y estructura. IV. Jurisdicción. V. Procedimiento ante la Corte. VI. Análisis de la jurisprudencia de la Corte. VII. Relación entre el Tribunal Africano y la Comisión. VIII. Conclusión y estado de la cuestión. IX. Bibliografía.
\end{abstract}

* Ha cursado el Bachillerato de Humanidades y Ciencias Sociales obteniendo la calificación de Matrícula de Honor y es Doble Graduado en Derecho y Administración y Dirección de Empresas por la Universidad de Sevilla (España). Colabora activamente con distintas ONG tanto españolas (Amnistía Internacional y Oficina de Derechos Sociales) como irlandesas (Kingsriver Community). Actualmente está cursando un Diploma de Especialización en Derechos Humanos y Democratización y forma parte de la Clínica de Derechos Humanos de Sevilla.

Este artículo forma parte de la tesis escrita por el mismo autor sobre El sistema regional africano de protección de los derechos humanos: especial referencia a sus instituciones jurisdiccionales.

Artículo recibido el 13 de junio de 2016 Aprobado para publicación el 22 de septiembre de 2016 
RESUMEN: En el presente artículo analizaremos unos de los tribunales regionales de protección de los derechos humanos más desconocidos en el mundo occidental: el Tribunal Africano de Derechos Humanos y de los Pueblos (TADHP). Si bien fue creado en 2004, no ha sido sino en los últimos años cuando ha llevado a cabo una incontestable tutela de los derechos humanos en África, actitud que ha dado lugar a que, pese a los arduos retos que aún ha de afrontar, tanto la sociedad civil como organismos pro derechos humanos y académicos hayan puesto sus esperanzas en esta institución en aras de acabar con la impunidad que azota dicho continente, siguiendo los pasos de sus continentes hermanos: América y Europa.

Palabras clave: Derechos Humanos en África, TADHP, Comisión Africana, Jurisprudencia Africana.

ABSTRACT: In this article we will analyze one of the less known regional courts of human rights protection to the western world: the African Court on Human Rights and People's Rights (AfCHPR). Although it was created in 2004, it was not until recent years that it has irrefutably protected human rights in Africa. This attitude has caused, despite the difficult challenges to be faced, that both the civil society as well as pro human rights and academic bodies to put their hope in this institution in order to end the impunity that reigns in said continent, following the steps of its sibling continents: America and Europe.

Key words: Human rights in Africa, AfCHPR, African Commission, African Binding Legal precedent.

RÉSUMÉ: Le présent article permet de se familiariser avec une des cours régionales de justice au service de la protection des êtres humains les moins connues dans le monde occidental: la Cour africaine des droits de l>homme et des peuples (CAfDHP). Bien que créée en 2004, ce n'est qu'au cours des dernières années qu'elle s'est incontestablement attachée à défendre les droits humains en Afrique. Si elle doit encore faire face à de nombreux défis, il n'en demeure pas moins qu'une telle attitude permet à la société civile comme à des organisations de défense de citoyens ou du monde universitaire de placer leurs espoirs dans cette institution afin d'en finir avec l'impunité qui étrangle ce continent pour que l'Afrique puisse ainsi suivre le chemin jadis emprunté par l'Europe et l'Amérique.

Mots-clés: Droits des êtres humains en Afrique, CAfDHP, Cour Africaine, Jurisprudence africaine. 


\section{INTRODUCCIÓN}

En 1998 fue adoptado por la Asamblea de Jefes de Estado y de Gobierno de la Organización para la Unidad Africana (OUA) el Protocolo a la Carta Africana sobre el establecimiento de un Tribunal Africano de Derechos Humanos y de los Pueblos (Tribunal), produciéndose de esta forma un avance sustancial en la consolidación del Sistema Regional Africano de Derechos Humanos.

Ya en la sesión constitutiva del Tribunal se declaró la importancia que implicaría la creación de dicho órgano en el continente africano, complementando el mandato de la Comisión Africana y fortaleciendo la protección de los derechos humanos y de los pueblos. Así pues, en opinión de autores como Saavedra Sánchez: "En el contexto africano, la Corte se establece como un mecanismo a través del cual se pretende hacer los derechos humanos plenamente exigibles". ${ }^{1}$

En el presente artículo comezaremos analizando el origen histórico y la composición y estructura del Tribunal, para a continuación proceder al estudio de su jurisdicción, así como del procedimiento a seguir ante la Corte. El examen de dichas cuestiones nos servirá de preludio para adentrarnos en una de las cuestiones más interesantes del Sistema Regional Africano: la jurisprudencia emanada de la Corte y la relación entre el Tribunal y la Comisión. Todo ello nos permitirá tener una visión global del Sistema Africano vigente de derechos humanos y del funcionamiento de sus instituciones jurisdiccionales.

\section{GÉNESIS DEL TRIBUNAL AFRICANO}

Si bien durante el proceso de elaboración de la Carta de Banjul determinadas voces se alzaron a favor de la creación de un Tribunal Africano facultado

1 Véase Saavedra Álvarez, Yuria, "El sistema africano de derechos humanos y de los pueblos. Prolegómenos", Anuario Mexicano de Derecho Internacional, vol. 8, 2008, p. 703. 
Esta revista forma parte del acervo de la Biblioteca Jurídica Virtual del Instituto de Investigaciones Jurídicas de la UNAM

para emitir decisiones vinculantes, ${ }^{2}$ el peso de la cultura africana, donde prevalecen los acuerdos amistosos sobre la litigación ante un tercero, junto con la defensa por parte de los gobiernos africanos del principio de soberanía y de no injerencia en los asuntos internos, motivaron que sólo se previera la Comisión Africana como mecanismo de salvaguarda de la Carta Africana.

No obstante, en la década de los noventa diversos factores, entre los que destacan "la emergencia y la consolidación del Estado de Derecho en África, la debilidad de la Comisión, la evolución de la actitud de los Estados africanos hacia la jurisdicción internacional así como la creación del Tribunal Penal Internacional para Ruanda", ${ }^{3}$ propiciaron retomar la idea de crear un Tribunal Africano que completara el sistema.

Se inició así un debate, donde tuvieron cabida juristas, expertos gubernamentales, profesores y distintas ONG, acerca de la supresión de la Comisión y la transferencia de sus funciones al futuro tribunal u optar por crear una institución jurisdiccional que complementara y fortaleciera las funciones de aquella. Finalmente se abogaría por la segunda opción.

Al igual que ocurriera en el proceso de adopción de la Carta Africana, destaca el papel desempeñado por la Comisión Internacional de Juristas, institución que desde un inicio reclamó la necesidad de constituir una Corte Africana para hacer frente a la violación de derechos humanos que habían tenido lugar en África durante la década de los setenta y ochenta. De hecho, fue esta institución la que llevó a cabo en 1993 el primer borrador sobre la creación de un Tribunal Africano de Derechos Humanos.

2 En la Resolución adoptada en la Conferencia de Lagos en 1961, conocida como "Ley de Lagos”, considerada por los autores como el punto de partida de la Carta Africana, ya se reconoció por parte de los intervinientes la necesidad de establecer una Corte Africana de Derechos Humanos. No obstante, se abandonó esta pretensión en aras de evitar controversias entre los Estados que pusiera en peligro la adopción de la Carta Africana. Al respecto, en opinión de M’Baye, considerado uno de los padres de la Carta Africana: “Au moment de l'adoption de la Charte, l'expérience prouvait que l'instauration d'une Cour des droits de l'homme en Afrique était peut-étre prématurée. Il faut se souvenir que le droit africain traditionnel fait une place peu importante á la procedure contentieuse. Le droit processuel africain est essentiellement conciliatoire. Néanmoins le moment est venu de revenir sur la question”. Véase Keba, M’Baye, "Les Droits de L'homme En Afrique”, Revue Internationale de Droit Comparé, vol. 45, núm. 3, 1993.

3 Véase San Martín Sanchez de Munián, Laura, "Comentarios acerca de la creación de un Tribunal Africano de Derechos Humanos y de los Pueblos", Anuario de Derecho Internacional, vol. 15, 1999, p. 513. 
Esta revista forma parte del acervo de la Biblioteca Jurídica Virtual del Instituto de Investigaciones Jurídicas de la UNAM

Este borrador fue presentado en 1994 ante la Organización para la Unidad Africana (OUA), adoptándose una resolución en la que por primera vez se ordenaba el mandato de crear una comisión de expertos para la elaboración de un Proyecto de Estatuto de la Corte y se reconocía "the need for the establishment of the Court to complement and reinforce the Commission" ante las graves violaciones de derechos humanos y de los pueblos producidas en el continente.

Esta resolución fue seguida por distintas reuniones donde se concretaron los extremos de la creación del nuevo Tribunal, destacando, en este sentido, la Conferencia de Cape Town (Sudáfrica) en 1995 y la Conferencia de Nouakchott (Mauritania) en abril de 1997. En esta última se elevó el requisito de ratificación para la entrada en vigor de la Corte de 11 a 15 Estados, se optó por la creación de una única sala y se estableció la necesidad de que los individuos y organizaciones no gubernamentales pudieran apelar ante la Corte siempre y cuando el Estado concernido lo autorizara previamente.

Tras estas dos reuniones, tuvieron que pasar ocho meses para que en la Asamblea mantenida en Addis Adeba ${ }^{4}$ (Etiopía) se adoptara el Protocolo definitivo, siendo aprobado en la trigésimo cuarta sesión ordinaria de la Asamblea General que tuvo lugar en junio de 1998, en Ouagadougou (Burkina Fasso). ${ }^{5}$

Finalmente, el Protocolo de la Carta Africana para la creación de una Corte Africana de Derechos Humanos y de los Pueblos (Protocolo) entró en vigor en enero de 2004, habiendo sido ratificado a fecha de mayo de 2016 por dieciséis Estados. ${ }^{6}$

En dicho Protocolo se encomienda al Tribunal la adopción de su propio Reglamento de Procedimiento, debiendo en su redacción consultar a la Comisión. ${ }^{7}$ Este mandato fue cumplido en 2010 con la elaboración

4 En el discurso de apertura de la Conferencia donde fue adoptó el Protocolo, el entonces Secretario General de la OUA, Salim Ahmed Salim, afirmó: "Human Rights is a basic requirement in any society and a pre-requisite for human progress and development". Véase "Talks Open in Addis Abeba on Establishing African Human Right Court”, Deutsche Presse-Agentur, available in Lexis, News Library, CURNWS File, December 12, 1997.

5 La Resolución adoptada puede ser consultada en: http://www.au.int/en/decisions (fecha de consulta: mayo de 2016)

6 Los Estados que han ratificado el Protocolo pueden ser consultados en: http: / /www. au.int/en/treaties/status (fecha de consulta: mayo de 2016).

7 Artículo 33, Protocolo. 
del Reglamento de Procedimiento Interno de la Corte (Reglamento). ${ }^{8}$ En 2007 la sede permanente de la Corte fue establecida en Arusha (Tanzania).

\section{COMPOSICIÓN Y ESTRUCTURA}

De conformidad con el Protocolo, la Corte Africana se compone de once jueces nacionales de Estados miembros de la Unión Africana ${ }^{9}$ (en adelante UA) ${ }^{10}$ Se exige que sean elegidos "in an individual capacity from among jurists of high moral character and of recognized practical, judicial or academic competence and experience in the field of human and peoples' rights". ${ }^{11}$

Para asegurar la representación geográfica, se imposibilita la coexistencia de dos jueces nacionales de un mismo Estado en la Corte. ${ }^{12}$ Los magistrados serán nombrados por la Asamblea de Jefes de Estado y de Gobierno de la UA en votación secreta de entre los tres candidatos propuestos por cada Estado miembro, ${ }^{13}$ teniendo en cuenta las principales regiones africanas y sus tradiciones jurídicas, así como una adecuada representación por género. ${ }^{14}$

8 Tanto el Protocolo de la Corte como su Reglamento Interno pueden ser objeto de consulta en: http://en.african-court.org/index.php/basic-documents/basic-documents-featured-articles (fecha de consulta: mayo de 2016).

9 En este sentido, el Protocolo sigue la línea del Convenio Americano (artículo 51) y no impone la elección de tantos jueces como Estados miembros. En cambio en el Convenio Europeo se establece que el Tribunal se compondrá de un número de jueces igual al de las Altas Partes Contratantes (artículo 20).

10 La Organización para la Unidad Africana (OUA) fue reemplazada en 2002 por la Unión Africana (UA). Actualmente de los 55 Estados que conforman el continente africano, tan solo Marruecos no es parte de la UA.

11 (1) Art. 11.1 Protocolo.

(2) Actualmente son magistrados de la Corte Africana Augustino S. L. Ramadhani (Tanzania), Angelo Vasco Matusse (Mozanbique), Elsie Nwanwuri Thompson (Nigeria), Gérard Niyungeko (Burundi), Fatsah Ouguergouz (Algeria), Duncan Tambala (Malawi), Sylvain Oré (Costa de Marfil), El Hadji Guisse (Senegal), Ben Kioko (Kenia), Rafaa Ben Achour (Túnez) y Solomy Balungi Bossa (Uganda).

12 Art. 11.2, Protocolo.

$13 \mathrm{Al}$ no recogerse expresamente en el Protocolo el número de votos requeridos para que un candidato sea elegido juez por la Asamblea de la UA, habría que acudir al Reglamento de Funcionamiento de la Asamblea, donde en su artículo 25 se establece que las decisiones se adoptarán por mayoría de dos tercios de todos los miembros presentes.

14 Artículos 12 y 14 del Protocolo y 13 del Reglamento. 
Esta revista forma parte del acervo de la Biblioteca Jurídica Virtual del Instituto de Investigaciones Jurídicas de la UNAM

Según el artículo 14 del Protocolo, el mandato de los jueces será de seis años, pudiendo ser reelegidos tan solo una vez. Asimismo, todos los jueces, a excepción del presidente, tienen encomendado su cargo a tiempo parcial. ${ }^{15}$

La independencia de los jueces se deberá garantizar de conformidad con el derecho internacional. A este respecto, se precisa que al desempeñar sus funciones, los jueces de la Corte disfrutarán de las inmunidades reconocidas a los agentes diplomáticos por el derecho internacional y éstos no podrán ser considerados responsables "at no time" de las opiniones o decisiones adoptadas en el ejercicio de sus funciones. Además, se prohíbe el ejercicio de actividades que puedan menoscabar su independencia e imparcialidad y se prevé la recusación del juez nacional del Estado que es parte en el asunto. ${ }^{16}$

Con respecto al procedimiento de remoción de los jueces, se exige el fallo unánime del resto de magistrados de la Corte, haciéndose definitiva la decisión una vez que sea aprobada por la Asamblea de Jefes de Estado y de Gobierno. ${ }^{17}$

En este sentido, el professor Nsongurua J. Udombana afirma: "as the experience of the Commission shows, however, while these measures are necessary, they are not sufficient to ensure institutional independence in the absence of other measures, such as sufficient funding and independent enforcement powers". ${ }^{18}$

15 A diferencia de la Corte Africana y de la Corte Interamericana (artículo 11, Reglamento de la Corte), el Tribunal Europeo de Derechos Humanos funciona de manera permanente (artículo 19, Convenio Europeo).

No obstante, en el caso africano se optó por esta opción para economizar recursos, en tanto que se alegó la no necesidad de disponer de un tribunal permanente durante los primeros años de su funcionamiento debido a la escasa carga de trabajo del mismo, contemplándose la posibilidad de que en un futuro pudiera ser modificada tal decisión (artículo 15.4, Protocolo).

16 (1) Artículo 17, 18 y 22 del Protocolo. Con respecto a la recusación, el Protocolo se diferencia de la Convención Americana, en la que atendiendo al artículo 55: "El juez que sea nacional de alguno de los Estados Partes en el caso sometido a la Corte, conservará su derecho a conocer del mismo".

(2) Cabe mencionar como ejemplo de recusación el caso Association Juriste d'Afrique v. Côte d'Ivoire en el que se establece: "conformément à l'article 22 du Protocole relatif à la Charte africaine des droits de l'homme et des peuples portant création d'une Cour africaine des droits de l'homme et des peuples, et à l'article 8.2 du Règlement intérieur de la Cour, Monsieur Sylvain ORE, membre de cette Cour, de nationalité ivoirienne, s'est récusé” (párr. 2).

17 Artículos 19 del Protocolo y 7o. del Reglamento. Tal sometimiento a la decisión ulterior de la Asamblea no estaba prevista en el Proyecto de Protocolo y ha sido objeto de críticas por los autores en tanto que puede socavar la independencia de la Corte.

18 Véase Udombana, Nsongurua, "Toward the African Court on Human and People' Rights: Better Late than Never", Yale Human Rights and Development Journal, vol 3, 2014, p. 85. 


\section{JURISDICCIÓN}

La jurisdicción del Tribunal aparece regulada en los artículos 3 a 9 del Protocolo. ${ }^{19}$ En opinión de la doctrina "Its jurisdictional provisions are the heart of the Protocol, as they determine who will have access to the Court, under what conditions, and what types of violations will be redressed". ${ }^{20}$

A continuación pasaremos al análisis de las tres funciones encomendadas al Tribunal Africano: función contenciosa, función consultiva y función arbitral.

\section{Jurisdicción contenciosa}

Respecto a la competencia ratio materiae, en el artículo tercero del Protocolo bajo la rúbrica "Jurisdicción" se establece: "The jurisdiction of the Court shall extend to all cases and disputes submitted to it concerning the interpretation and application of the Charter, this Protocol and any other relevant Human Rights instrument ratified by the States concerned". ${ }^{21}$

Los autores señalan la transcendencia de esta última cláusula en tanto que faculta al Tribunal para pronunciarse no sólo sobre instrumentos africanos como la Convención de la OUA sobre los Refugiados en África de 1969, la Carta Africana de los Derechos y Bienestar del Niño de 1990 o el Protocolo a la Carta Africana de los Derechos de la Mujer en África de 2003, entre otros, sino también posibilita la competencia del tribunal sobre instrumentos internacionales como el Pacto Internacional de Derechos Civiles y Políticos y el Pacto Internacional de Derechos Económicos, Sociales y Culturales. ${ }^{22}$

19 Siendo objeto de desarrollo por el Reglamento en la Sección III y V.

20 Ibidem, p. 86.

21 Esta cláusula debe ser leída junto con el artículo 7o. del Protocolo en el que se establece que "The Court shall apply the provision of the Charter and any other relevant human rights instruments ratified by the States concerned".

22 Al respecto, en el Caso Emmanuel Joseph Uko\& Others v. Republic of South Africa el demandante alega la violación del Pacto Internacional de Derechos Civiles y Políticos de 1966. En esta línea, en el caso Frank David Omary \& Others v. Tanzania el demandante invoca la violación 
Esta revista forma parte del acervo de la Biblioteca Jurídica Virtual del Instituto de Investigaciones Jurídicas de la UNAM

No obstante, la expresión "instrumentos relevantes" no ha sido objeto de una interpretación pacífica. Mientras que para autores como Udombana, Naldi y Magiveras la disposición extiende la competencia del tribunal a tratados regionales, subregionales, bilaterales, multilaterales e internacionales, destacando las positivas implicaciones de dicha cláusula, en tanto, "where a State tried to invoke a "clawback clause» to justify a breach of internationally protected rights, the vitim could simply invoke a treaty protecting the same rights, such as the ICCPR, that did not include a similar clawback clause", ${ }^{23}$ para autores como Heyns y Christof Heyns "si los casos pudieran ser llevados ante la Corte Africana de Derechos Humanos sobre la base de que, por ejemplo, los tratados de las Naciones Unidas han sido violados, sin referencia alguna a la Carta Africana, esto conduciría a un caos jurisprudencial y socavaría la naturaleza particular de la Carta Africana". ${ }^{24}$ Así pues, estos autores abogan por entender el término "relevante" como una acotación a los instrumentos africanos. No obstante, en este sentido aun persistiría la duda de si el Tribunal tendría competencia sobre los tratados de organizaciones subregionales africanas como la Comunidad Económica de Países del África Occidental (ECOWAS) o la Comunidad para el Desarrollo del África Austral (SADC).

Respecto a la ratio personae, el artículo 5o. del Protocolo faculta para dirigirse a la Corte y a la Comisión (i), al Estado parte que hubiera sometido un caso ante la Comisión (ii), al Estado parte demandado ante la Comisión (iii), al Estado parte de la nacionalidad de la víctima de una violación de los derechos humanos (iv) y a organizaciones intergubernamentales (v).

Además, los individuos y las ONG que tengan el estatuto de observador ante la Comisión pueden acudir a la Corte siempre que el Estado frente al cual se interpone la demanda emita una declaración de competencia con-

de la Declaración Universal de Derechos Humanos de 1948. Como ejemplo de no admisibilidad de la demanda por no entrar el asunto dentro de la ratio materiae del Tribunal, podemos citar el caso Efoua Mbozo'o Samuel v. Pan African Parliament donde la Corte Africana estableció que las alegaciones del demandante estaban basadas exclusivamente en una infracción del contrato de trabajo.

23 Véase Udombanat, Nsongurua J., Toward, cit., p. 85; Naldi, G. y Magliveras, K., "Reinforcing the African System of Human Rights: The Protocol on the Establishment of a Regional Court of Human and Peoples' Rights”, Netherlands Quarterly of Human Rights, 1998, p. 435.

24 Véase Heyns, Christof, "The African Regional Human Rights System: In Need of Reform?”, African Human Rights Law Journal, vol. 1, núm. 2, 2001, p. 119 
Esta revista forma parte del acervo de la Biblioteca Jurídica Virtual del Instituto de Investigaciones Jurídicas de la UNAM

forme al artículo 36.5. ${ }^{25}$ Por tanto, en el Protocolo se establece una jurisdicción automática para los casos presentados por la Comisión y por los Estados que hayan ratificado el Protocolo y una jurisdicción opcional para ONG e individuos. ${ }^{26}$

Esta decisión ha sido una de las más criticadas por los autores, así pues, el magistrado sudafricano Ducan Mlambo aboga por la modificación de dicho artículo, afirmando la importancia del acceso de los individuos a la Corte en tanto éstos son "the first target" en la violación de derechos humanos. En este sentido se pronuncia Harrington, quien establece: "The limitation on locus standi must be understood as a cynical move to diminish what power the Court might have over States by making it less accessible to those most likely to bring cases". ${ }^{27}$

No obstante, debemos señalar que los individuos y las ONG sí están facultados para acudir ante la Comisión, ${ }^{28}$ institución que conforme al mencionado artículo 5o. puede someter a su vez el caso a la Corte.

\section{Jurisdicción consultiva}

Atendiendo al artículo 4o. del Protocolo, ${ }^{29}$ la Corte podrá emitir un dictamen consultivo sobre cualquier materia jurídica relacionada con la Carta Africana, así como, sobre cualquier otro instrumento relevante de derechos

25 A mayo de 2016 siete Estados han emitido dicha Declaración de Competencia: Burkina Faso, Mali, Costa de Marfil, Ghana, Benín, Tanzania y Malawi.

26 No obstante cabe señalar que con relación a posibilidad de presentar casos ante la Corte Africana por parte de las ONG en el Protocolo se establece: "The Court may entitle relevant Non Governmental organizations (NGOs) with observer status before the Commission". A este respecto, algunos autores han llamado la atención sobre el término "relevant", precisando que se trata de un "misterio" el significado de dicho concepto, dado un poder discrecional a la Corte innecesario. Véase Kaguongo W., "The Questions of Locus Standi and Admissibility before the African Court on Human and Peoples' Rights", Judiciary Watch Report, 2006, p. 83.

27 Véase Wright, Rebecca, "Finding an Impetus for Institutional Change at the African Court on Human and Peoples' Rights”, Berkeley Journal of International Law, vol. 24, 2006, p. 479 .

28 Artículo 55 de la Carta Africana.

29 Para un análisis del artículo 4 del Protocolo y de la jurisdicción consultiva de la Corte, véase Van der Mei, Anne Pieter, "The Advisory Jurisdiction of the African Court on Human and Peoples' Rights”, African Human Rights Law Journal, vol 25, 2005. 
Esta revista forma parte del acervo de la Biblioteca Jurídica Virtual del Instituto de Investigaciones Jurídicas de la UNAM

humanos, a petición de un Estado miembro de la UA ${ }^{30}$ (i), la Unión Africana (ii), alguno de sus órganos (iii), o cualquier otra organización africana reconocida por la UA (iv). ${ }^{31}$

No existe unanimidad entre los autores sobre qué debe entenderse por "any African organization recognized by the $O A U$ ". Mientras que para autores como Udombana podría referirse a organizaciones subregionales como ECOWAS, para Mbondenyi podría hacer referencia a las ONG a las que se les haya reconocido el estatuto de observador ante la Comisión.

En tanto, la Comisión también tiene atribuidas competencias consultivas, en aras de evitar interpretaciones discrepantes, se precisa que la Corte no puede emitir su juicio sobre una materia que esté siendo objeto de examen por la Comisión. ${ }^{32}$

El Tribunal expondrá las razones de sus opiniones consultivas, estando facultado todo juez para formular opinión separada. ${ }^{33}$ Las opiniones del Tribunal, al igual que en el sistema europeo y americano, no son vinculantes, pero "en la práctica tales opiniones pueden constituir un elemento importante para la interpretación dinámica de las Convenciones sobre derechos humanos". ${ }^{34}$

Autores como Ougande señalan la importancia de la jurisdicción consultiva de la Corte, precisando al respecto que las opiniones emitidas por el Tribunal contribuirán a la consolidación del Estado de derecho en la cultura africana y enriquecerán la jurisprudencia de la Corte. ${ }^{35}$

A junio de 2016 el Tribunal Africano de Derechos Humanos y de los Pueblos ha emitido seis opiniones consultivas, las cuales serán objeto de análisis en un epígrafe posterior de este artículo.

30 Nótese que no se exige la ratificación del Protocolo para que un Estado miembro de la UA pueda solicitar opiniones consultivas ante la Corte.

31 En el artículo 68 del Reglamento se precisa: "The request shall be on legal matters and shall state with precision the specific questions on which the opinion of the Court is being sought".

32 La competencia otorgada al Tribunal Africano en materia consultiva es similar a la ostentada por la Corte Interamericana de Justicia. En cambio, en el Convenio Europeo se establece una competencia consultiva más restrictiva: "ElTribunal podrá emitir opiniones consultivas, a solicitud del Comité de Ministros, acerca de cuestiones jurídicas relativas a la interpretación del Convenio y de sus Protocolos" (artículo 47.1).

33 Artículos 4.2 Protocolo y 73.2 Reglamento

34 Véase San Martín Sanchez de Munián, op. cit., p. 522.

35 Véase African Legal Aid (AFLA), "Introducing the New African Court on Human and Peoples' Rights", Africa Legal Aid Quarterly, 2006, p.6. 
Esta revista forma parte del acervo de la Biblioteca Jurídica Virtual del Instituto de Investigaciones Jurídicas de la UNAM

\section{Jurisdicción arbitral}

Reflejo de la cultura africana donde los acuerdos amistosos prevalecen sobre el sometimiento de la disputa a un tercero, en el artículo 9o. del Protocolo se faculta a la Corte para tratar de alcanzar un acuerdo amistoso en un caso sometido a su jurisdicción.

Tanto en el Protocolo como en el Reglamento no se hace mención bajo qué condiciones las partes pueden solicitar un procedimiento de conciliación. Si bien, en la redacción del precepto se utiliza la expresión "The Court may try" por lo que en principio quedaría a discrecionalidad de la Corte.

En el artículo 57 del Reglamento se establece la confidencialidad de las negociaciones para llegar a un acuerdo amistoso, precisándose al respecto: "no written or oral communication and no offer of concession made as part of such negotiations shall be mentioned or referred to in the proceedings before the Court".

En opinión de autores como Mbondenyi, se trata de un desacierto otorgar competencia arbitral a la Corte pues la propia Comisión tiene atribuida tal función. Así pues, el autor propone "where the parties to a dispute agree to resolve it amicably, the court may transfer the matter to the commission for settlement. Accordingly, this would save the court's time and also that of the parties". ${ }^{36}$

\section{Procedimiento ANTE LA CORTE AFRICANA}

El Protocolo establece las directrices generales relativas al procedimiento que ha de seguirse ante la Corte, especialmente sobre admisibilidad de los casos (i), instrucción y juicio (ii), alcance de las decisiones del Tribunal (iii) y reparaciones (iv), siendo desarrolladas tales disposiciones en la Sección IV del Reglamento de Funcionamiento Interno de la Corte.

\section{Admisibilidad y consideración de los casos}

Atendiendo al artículo 6.2 del Protocolo, la Corte deberá determinar la admisibilidad de los casos teniendo en cuenta las mismas reglas establecidas en la Carta Africana para la aceptación de los comunicados por la Comisión, entre las que destacan: la no utilización de un lenguaje despectivo o

36 Véase Mbondenyi, Morris Kiwinda, "Investigating the Challenges in Enforcing International Human Rights Law in Africa: Towards an Effective Regional System”, University of South Africa, Pretoria, 2006, p. 376. 
Esta revista forma parte del acervo de la Biblioteca Jurídica Virtual del Instituto de Investigaciones Jurídicas de la UNAM

insultante, la identificación de sus autores, el agotamiento de los recursos internos, siempre que no esté teniendo lugar un retraso injustificado en su resolución, y su presentación dentro de un periodo de tiempo razonable a partir del momento en que se agotaron dichos recursos internos. ${ }^{37}$ Con respecto a este último requisito, en el caso Mtikila \&Others v Tanzania at the African Court on Human and Peoples' Rights el Tribunal Africano determinó que un año no era un periodo irrazonable de tiempo.

En relación con los casos presentados por individuos y ONG, la Corte antes de decidir sobre su admisibilidad puede solicitar la opinión de la Comisión. Asimismo, una vez admitidos, se faculta al Tribunal a remitir los mismos ante la Comisión. Esta disposición ha sido vista por algunos autores como un movimiento innecesario de casos, donde queda reflejado la ineficacia de un sistema basado en dos instituciones que tienen encomendadas en gran medida las mismas funciones, ${ }^{38}$

\section{La instrucción y el juicio}

El procedimiento ante el tribunal será público, no obstante, bajo determinadas circunstancias recogidas en el Reglamento podrán celebrarse audiencias privadas. ${ }^{39}$ En el curso del proceso, las partes tienen derecho a ser representadas, pudiendo contar con asistencia jurídica gratuita si "el interés de la justicia lo requiere”, por lo que habrá que atender a la interpretación que la Corte haga de este concepto. Además, "se asegurará la protección y las facilidades necesarias, conforme al Derecho internacional, a todas las personas, testigos o representantes de las partes, llamadas a comparecer ante el Tribunal”. ${ }^{40}$

37 Otras de exigencias para la admisibilidad de los comunicados por la Comisión son: la compatibilidad de la petición con el Acta Constitutiva de la UA o con la Carta Africana; no estar basadas exclusivamente en noticias difundidas por los medios de comunicación; y no tratar de casos que ya han sido solucionados por los Estados implicados de conformidad con los principios de la Carta de las Naciones Unidas, la Carta de la Organización para la Unidad Africana o las disposiciones de la presente Carta (artículos 56 de la Carta Africana y 6o. del Protocolo).

38 Véase Mbondenyi, op. cit., p. 378.

39 A este respecto, en el artículo 43 Reglamento se establece: "the Court may, of its own accord or at the request of a party, hold its hearings in camera if, in its opinion, it is in the interest of public morality, safety or public order to do so".

40 Artículo 10 Protocolo, véase San Martín Sanchez de Munián, op. cit., p. 523. 
Al ser pública la audiencia ante la Corte, se ha conseguido avanzar en transparencia con respecto a la Comisión puesto que para esta institución se exige la confidencialidad de su actuación hasta que la Asamblea de Jefes de Estado y de Gobierno decida lo contrario. ${ }^{41}$

Conforme al artículo 26 del Protocolo, el Tribunal escuchará los alegatos de todas las partes, pudiendo ordenar la apertura de una investigación si así lo considera necesario. La Corte podrá aceptar tanto pruebas escritas como visuales, así como el testimonio de expertos, adoptando su dictamen sobre la base de dichas pruebas. Asimismo, se exige que los Estados parte colaboren con el Tribunal para facilitar el ejercicio de su cometido.

A este respecto, ciertos autores, si bien remarcan la importancia de esta última cláusula, dudan de su cumplimiento si no va acompañada de sanciones concretas en caso de desobediencia por parte de los Estados que se nieguen a colaborar. ${ }^{42}$

Al igual que en el sistema americano y europeo, la Corte puede adoptar medidas provisionales cuando sea necesario evitar un daño irreparable y en casos de extrema gravedad o urgencia. ${ }^{43}$ Sin embargo, a diferencia de la naturaleza reconocida a las sentencias, el Protocolo guarda silencio sobre si tales medidas provisionales son obligatorias, "por lo que su carácter vinculante no es muy obvio”. ${ }^{44}$ La Corte acordó la adopción de medidas provisionales por primera vez en el Caso de Libia, que será objeto de estudio en el siguiente epígrafe.

\section{Alcance de las decisiones del Tribunal}

La Corte acordará el fallo por mayoría de sus miembros, estando facultados los jueces que no compartan las decisiones adoptadas por la mayoría de la Corte a expresar su opinión divergente. ${ }^{45}$ La sentencia no puede ser objeto

\footnotetext{
41 Artículo 59 de la Carta Africana.

42 Ibidem, p. 523.

43 Artículos 27.2 del Protocolo y 51 del Reglamento

44 Véase Saavedra Álvarez, op. cit., p. 707.

45 Artículos 28.4 y 7 del Protocolo.
} 
de recurso, no obstante, el Tribunal puede revisar su veredicto si nuevas pruebas aparecen. ${ }^{46}$ La sentencia será interpretada por el propio Tribunal. ${ }^{47}$

A diferencia de la Comisión, en el Protocolo se establece un máximo de noventa días durante los cuales el Tribunal debe completar sus deliberaciones y se exige que el fallo de la Corte sea motivado, evitándose así la práctica seguida por la Comisión en sus primeros pronunciamientos donde éstos no eran razonados ni detallados.

En cuanto a la naturaleza de la sentencia, no se trata de un fallo meramente declaratorio en el que se precisa que un Estado ha incurrido en la violación de derechos humanos, sino que se reconoce su carácter vinculante. Así pues, atendiendo al artículo 30 del Protocolo, "los Estados parte se comprometen a acatar las decisiones del Tribunal y a asegurar su ejecución en el plazo fijado por éste". ${ }^{48}$

En caso de incumplimiento de la sentencia, el Consejo de Ministros de la UA en nombre de la Asamblea recibe el mandato de garantizar la ejecución de la misma.

A este respecto debemos acudir al artículo 23.2 del Acta Constitutiva de la UA donde se establece: "Any Member State that fails to comply with the decisions and policies of the Union may be subjected to other sanctions, such as the denial of transport and communications links with other Member States, and other measures of a political and economic nature to be determined by the Assembly".

En opinión de los autores, los mecanismos que se contemplan para la ejecución de la sentencia son muy limitados, más cuando la experiencia de la Comisión ha demostrado la reticencia de la mayoría de Estados africanos a cumplir con las decisiones adoptadas.

Si bien, a diferencia de la Comisión, se prevé como medio de presión, que la Corte, en el Informe de Actividades Anual que cada año remita a la Asamblea, pueda especificar los Estados que no han cumplido con las resoluciones aprobadas. ${ }^{49}$

46 Artículos 28.2 y 3 del Protocolo.

47 Cabe mencionar que el quórum para la válida constitución de la Sala es de al menos siete magistrados. Artículos 23 del Protocolo y 17 del Reglamento.

48 Véase San Martín Sanchez de Munián, op. cit., p. 524.

49 Artículos 29.2 y 31 del Protocolo 
Esta revista forma parte del acervo de la Biblioteca Jurídica Virtual del Instituto de Investigaciones Jurídicas de la UNAM

\section{Reparaciones}

La previsión de las reparaciones es otra de las novedades introducidas en el Protocolo con respecto a la Carta Africana. Así, en el artículo 27.1 del mismo se establece: "If the Court finds that there has been violation of a human or peoples' rights, it shall make appropriate orders to remedy the violation, including the payment of fair compensation or reparation".

Añadiéndose en el artículo 63 del Reglamento: "The Court shall rule on the request for the reparation, submitted in accordance with Rule 34 (5) of these Rules, by the same decision establishing the violation of a human and peoples' right or, if the circumstances so require, by a separate decisión".

Algunos autores señalan que los términos "compensación" y "reparación" están formulados de manera ambigua e imprecisa, siendo importante evitar "a restrictive interpretation of its remedial powers by ordering adequate reparation whenever the interest of justice demands". ${ }^{50}$

Con respecto a las costas judiciales, el Protocolo guarda silencio y atendiendo al Reglamento de la Corte se establece que a menos que el Tribunal establezca lo contrario, cada parte deberá soportar sus propias costas. ${ }^{51}$ Por lo tanto, en principio parece que queda a su discrecionalidad la condena en Costas. ${ }^{52}$

Para concluir este epígrafe citaremos al profesor Morris Kiwinda Mbondenyi quien al respecto precisa:

The African Court has more potential to contribute to the effective enforcement of human rights in Africa than its counterpart, the commission. [Nevertheless] in order to be persuasive, the court may have to be innovative in the presentation of its judgements. Mechanisms such as indicating both sides of the argument, giving ratio decidendi, dealing with all points raised and examining issues of admissibility and jurisdiction fully and properly, are essential for its own legitimacy. ${ }^{53}$

50 Véase Mbondenyi, op. cit., p. 384.

51 Artículo 30 del Reglamento de la Corte.

52 Cabe mencionar que con frecuencia el Tribunal impone a cada parte el pago de sus costas como principio general. Tal regla es seguida en las sentencias Appl. No 006/2013 Wilfred Onyango \& Others v.United Republic of Tanzania, Appl.No 004/2013 - Lohé Issa Konaté v. Burkina Faso, entre otras. Esto puede llevar a desincentivar a las víctimas de violaciones de derechos humanos a presentar casos ante la Corte Africana, pues aún venciendo en el juicio van a tener que pagar las costas del mismo.

53 Ibidem, p. 385. 


\section{ANÁLISIS DE LA JURISPRUDENCIA DE LA CORTE}

La historia del Tribunal Africano se caracteriza por su lento proceso de implementación. Si la Carta Africana fue aprobada en 1981, para la redacción del Protocolo por el que se crea la Corte Africana de Derechos Humanos y de los Pueblos se esperó hasta 1998, produciéndose su entrada en vigor seis años más tarde. Asimismo, hasta 2006 los magistrados no fueron elegidos y hasta 2008 la Corte no dispuso de una sede permanente en Tanzania.

Va a ser en dicho año cuando le sea presentado al Tribunal su primer caso Appl.No 001 /2008- Michelot Yogogombaye versus the Republic of Senegal, teniendo lugar su histórica primera sentencia el 15 de diciembre de 2009.

\section{Primeros pronunciamientos de la Corte: Caso Yogogombaye vS. Senegal}

En dicho caso, el demandante Michelot Yogogombaye, nacional del Chad y residente en aquel momento en Bienne (Suiza), interpuso una demanda ante el Tribunal contra la República de Senegal, donde se solicitaba la suspensión de los procedimientos en vigor iniciados por el Estado de Senegal con el objeto de procesar al antiguo jefe de Estado de Chad, Hissein Habré, asilado político durante ocho años en Dakar (Senegal).

El señor Yogogombaye alegó que Hissein Habré fue considerado sospechoso en el año 2000, de complicidad en los crímenes contra la humanidad, crímenes de guerra y actos de tortura en el ejercicio de sus tareas como jefe del Estado, una alegación basada en las quejas de presuntas víctimas originarias del Chad. ${ }^{54}$

Asimismo, expuso que, en violación del "sacrosanto" principio de irretroactividad penal, la República de Senegal adoptó una ley reformando la Constitución y autorizando la aplicación retroactiva de sus leyes penales con el objetivo de procesar exclusiva y únicamente al señor Hissein Habré. ${ }^{55}$

Por todo ello, el solicitante concluyó que había tenido lugar la violación de varias cláusulas del Preámbulo y de los artículos de la Carta Africana y que se había producido un abuso del principio de jurisdicción universal, al

54 Michelot Yogogombaye versus the Republic of Senegal, párrafo 18.

55 Ibidem, párrafo 20. 
Esta revista forma parte del acervo de la Biblioteca Jurídica Virtual del Instituto de Investigaciones Jurídicas de la UNAM

existir una motivación política y pecuniaria por parte del Estado de Senegal. Así pues, solicitó al Tribunal:

Ordenar a la República y Estado de Chad y a la República y Estado de Senegal la creación de una Comisión Nacional para Chad de la "Verdad, Justicia, Reparaciones y Reconciliación” para todos los crímenes cometidos en Chad entre 1962 y 2008; y al hacerlo resolver a la manera Africana el problemático asunto del antiguo Jefe del Estado del Chad, Hissein Habré. ${ }^{56}$

Por su parte, el Estado de Senegal declaró no haber emitido la declaración de competencia necesaria para que la Corte pueda conocer de las demandas interpuestas por individuos y se opuso a las alegaciones de presunta violación del principio de irretroactividad penal.

El Tribunal ante el caso planteado invocó los preceptos 5.3 y 34.6 del Protocolo en los que se establece respectivamente:

The Court may entitle relevant Non Governmental organizations (NGOs) with observer status before the Commission, and individuals to institute cases directly before it, in accordance with article 34 (6) of this Protocol.

At the time of the ratification of this Protocol or any time thereafter, the State shall make a declaration accepting the competence of the Court to receive cases under article 5 (3) of this Protocol. The Court shall not receive any petition under article 5 (3) involving a State Party which has not made such a declaration.

Atendiendo a la lectura conjunta de estos dos artículos, el Tribunal determinó que efectivamente carecía de jurisdicción para conocer de la demanda interpuesta, en tanto que el Estado de Senegal no había efectuado una declaración aceptando la competencia de la Corte para recibir los casos presentados por individuos y ONG. ${ }^{57}$

No obstante, el Tribunal interpretó que en la segunda frase del artículo 34.6 según la cual el "Tribunal no recibirá ningún caso de los contemplados en el artículo 5.3 que afecte a un Estado parte que no haya efectuado dicha

56 Ibidem, párrafo 23.6.

57 Con respecto a las costas procesales, el demandante solicitó el beneficio de la justicia gratuita y el demandado que sus costas fueran a cargo del señor Michelot Yogogombe. Finalmente, el Tribunal decidió aplicar la regla general establecida en el artículo 30 del Reglamento: "A no ser que se disponga otra cosa, cada parte se hará cargo de sus propias costas". 
Esta revista forma parte del acervo de la Biblioteca Jurídica Virtual del Instituto de Investigaciones Jurídicas de la UNAM

declaración", "el término «recibir» no debiera ser entendido sin embargo en su acepción literal como refiriéndose a «recibir físicamente» ni en su sentido técnico como refiriéndose a la «admisibilidad»" ${ }^{58}$ En otras palabras, la Corte determinó que atendiendo a dicho artículo nada impide al Tribunal recibir físicamente tales peticiones provenientes de individuos y ONG. ${ }^{59}$

Cabe destacar la opinión particular emitida por el juez Fatsah Ouguerouz, quien precisó al respecto que, pese a no ser de aplicación en el caso que nos concierne pues Senegal rechazó desde un primer momento la competencia de individuos para presentar casos ante la Corte, de la lectura de los mencionados preceptos, el Estado puede proceder a emitir tal declaración de competencia en la propia respuesta a la demanda interpuesta o incluso el Tribunal puede conocer del asunto si así se deduce de la actuación del Estado mediante la aplicación del principio del fórum prorrogatum. ${ }^{60}$

De esta forma, el juez Fatsah Ouguerouz, a través de una interpretación innovadora del artículo 34.6 del Protocolo, abre una nueva vía ante las limitaciones de presentar casos por parte de individuos y ONG ante la Corte.

\section{Decisiones significactivas: Christopher R. Mtikila vs. The United Republic of Tanzania}

Si bien desde su instauración hasta 2010 la Corte va a centrarse en mayor medida en solventar cuestiones de tipo administrativo y procedimental que en impartir justicia, emitiendo únicamente durante este periodo el pronunciamiento que ha sido objeto de análisis en líneas anteriores, a partir de 2011 parece que la Corte comienza a estar capacitada para hacer frente a su cometido, pronunciándose hasta mayo de 2016 sobre 25 casos. ${ }^{61}$

58 Ibidem, párrafo 39.

59 Véase C. Jalloh, Charles, "International Decision: Michelot Yogogombaye v. The Republic of Senegal.” American Journal of International Law, vol 104, núm. 4, 2010, p. 624.

60 El juez Fatsah Ouguerouz se expresa en los siguientes términos: «This consent may be expressed before the filing of an application against the State Party, with the submission of the declaration mentioned in Article 34 (6) of the Protocol. It may also be expressed later, either formally through the filing of such a declaration, or informally or implicitly through forum prorogatum», Opinión Particular Michelot Yogogombaye versus the Republic of Senegal, Párr 31

${ }^{61}$ Nótese que similar situación tuvo lugar al momento de constituir la Corte Interamericana. Constituida en 1980, hubo de esperarse seis años para su primer pronunciamiento, teniendo lugar el segundo cuatro años más tarde. 
Esta revista forma parte del acervo de la Biblioteca Jurídica Virtual del Instituto de Investigaciones Jurídicas de la UNAM

Al igual que la primera sentencia dictada por la Corte, los casos que le siguen no van a entrar a conocer del fondo del asunto. Así pues, en el asunto Youssef Ababou v. The Kingdom of Morocco ${ }^{62}$ donde el demandante Youssef Ababou había alegado que el Reino de Marruecos desestimó en repetidas ocasiones proporcionarle la documentación solicitada, entre la que se incluía el pasaporte y el documento nacional de identidad, pese a poder probar el cumplimiento de todos los requisitos necesarios para obtenerlos, el Tribunal determinó que no era competente para conocer de la demanda interpuesta, en tanto que el Reino de Marruecos no era Estado miembro de la Unión Africana y, por ende, no puede suscribir el Protocolo de la Corte Africana de Derechos Humanos.

Igual veredicto se establece en el asunto Daniel Amare and Mulugeta Amare v. Republic of Mozambique \& Mozambique Airlines ${ }^{63}$ donde los demandantes alegan que pese a contratar un vuelo de Maputo (Mozambique) hasta Nairobi (Kenia), el avión aterrizó en Pempa (Mozambique), región en la que fueron sometidos a torturas, confiscación del pasaporte, sustracción de una cantidad de $\$ 1000$ y, pese a contar con la documentación en regla, deportados a Tanzania. En Tanzania fueron retornados de nuevo a Pempa, donde finalmente las autoridades mozambiqueñas acordaron repatriarlos a Etiopía. La Corte precisó que no era competente para conocer del asunto en tanto Mozambique no había emitido la declaración de competencia de acuerdo con el artículo 34.6 del Protocolo.

Habría que esperar hasta el 14 de junio de 2013 para que la Corte dictara la primera sentencia que entrara a conocer del fondo del asunto, en el caso Christopher R. Mtikila v. The United Republic of Tanzania ${ }^{64}$ Pronunciándose el Tribunal en una resolución posterior del 13 de junio de 2014 sobre las reparaciones alegadas.

Para autores como Oliver Windridge nos encontramos ante " a watershed case", en tanto "this case contains many firsts - it is the first case to be considered on its merits, the first finding in favour of the applicant and the first matter to consider the issue of compensation and reparations" ${ }^{65}$

62 Véase Appl.No 007/2011 Youssef Ababou v. The Kingdom of Morocco.

63 Véase Appl.No 005/2011 Daniel Amare and Mulugeta Amare v. Republic of Mozambique \& Mozambique Airlines.

64 Véase Appl.No 011/2011 Rev. Christopher R. Mtikila v. The United Republic of Tanzania.

65 Véase Windridge, Oliver, “A Watershed Moment for African Human Rights: Mtikila \& Others v Tanzania at the African Court on Human and Peoples' Rights", Oxford Human Rights 
Esta revista forma parte del acervo de la Biblioteca Jurídica Virtual del Instituto de Investigaciones Jurídicas de la UNAM

Los hechos apreciados se remontan a 1992, fecha en la que tuvo lugar una reforma de la Constitución Tanzana en virtud de la cual se establece como condición sine qua non para presentarse como candidato a las elecciones presidenciales, parlamentarias y locales estar afiliado a un partido político y ser propuesto por éste. Desde dicha fecha, el demandante Christopher R. Mtikila, nacional de Tanzania, procedió durante más de quince años a impugnar la constitucionalidad de tal precepto.

Si bien, el Tribunal Supremo Tanzano falló a favor del peticionario, en 2010 la Corte de Apelación revocó el fallo, estableciendo que se trataba de un asunto esencialmente de naturaleza política y que, por ende, le correspondía al Parlamento su esclarecimiento.

Tras agotar los recursos internos, en junio de 2011 el demandante Christopher R. Mtikila procedió a presentar el caso ante el Tribunal Africano de Derechos Humanos y de los Pueblos, alegando la violación por la República de Tanzania del derecho de libre asociación (artículo 10 de la Carta Africana), el derecho a participar en los asuntos públicos y de gobierno (artículo 13.1 de la Carta Africana) y el principio de no discriminación (artículo 2o. de la Carta Africana). ${ }^{66}$ Asimismo, declaró la violación de las reglas del Estado de Derecho y la vulneración de los artículos 21 y 25 del Pacto Internacional de Derechos Civiles y Políticos. ${ }^{67}$

Por su parte, la República de Tanzania justificó la reforma constitucional en aras de salvaguardar la gobernanza y unidad del país, el orden público y la defensa nacional. Asimismo, presentó el Caso Castañeda Gutman vs. Mexico

Hub, 2015, disponible en: http://humanrights.dev3.oneltd.eu/a-watershed-case-for-african-hu man-rights-mtikila-and-others-v-tanzania/ (fecha de consulta: junio de 2016).

66 El demandante alegó para secundar su postura la comunicación de la Comisión núm. 211/98 Legal Resources Foundation v Zambia Fourteenth Activity Report en la que en el párrafo 64 establece: "Any measure which seeks to exclude a section of the citizenry from participating in the democratic processes is discriminatory and falls foul of the Charter".

67 En el artículo 25 se declara: “Todos los ciudadanos gozarán, sin ninguna de la distinciones mencionadas en el artículo 2, y sin restricciones indebidas, de los siguientes derechos y oportunidades: a) Participar en la dirección de los asuntos públicos, directamente o por medio de representantes libremente elegidos". Por su parte, en el artículo 21: "Se reconoce el derecho de reunión pacífica. El ejercicio de tal derecho sólo podrá estar sujeto a las restricciones previstas por la ley que sean necesarias en una sociedad democrática, en interés de la seguridad nacional, de la seguridad pública o del orden público, o para proteger la salud o la moral públicas o los derechos y libertades de los demás”. 
Esta revista forma parte del acervo de la Biblioteca Jurídica Virtual del Instituto de Investigaciones Jurídicas de la UNAM

para explicar que la decisión de prohibir candidaturas independientes depende de la realidad histórica de un Estado y de sus necesidades sociales. ${ }^{68}$

Ante los hechos alegados, la Corte procedió a acordar la admisibilidad del caso en tanto que Tanzania había emitido la declaración de competencia en favor de individuos y ONG, pudiendo entrar a conocer del fondo del asunto. A este respecto, el Tribunal comenzó invocando el artículo 13 de la Carta Africana: "(1) Every citizen shall have the right to participate freely in the government of his country, either directly or through freely chosen representatives in accordance with the provisions of the law.

(2) Every citizen shall have the right of equal access to the public service of his country".

Para a continuación precisar que todos los derechos reconocidos en el artículo 13 son derechos individuales, no derechos reconocidos a un grupo. Por tanto, la exigencia de estar afiliado a un partido político para presentarse como candidato no encuentra respaldo en la Carta de Banjul. ${ }^{69}$

La Corte estableció que si bien los derechos reconocidos en la Carta Africana pueden ser limitados tal y como se establece en el artículo 27 de la misma, el Tribunal recordó que las restricciones al ejercicio de los derechos deben ser relevantes, suficientes y razonablemente proporcionales al objetivo legítimo perseguido, ${ }^{70}$ concluyendo en su razonamiento que "in any event, the restriction on the exercise of the right through the prohibition on independent candidacy is not proportionate to the alleged aim of fostering national unity and solidarity". ${ }^{71}$

Asimismo, la Corte sostuvo que la medida adoptada por el Estado de Tanzania había supuesto una violación de la libertad de asociación estable-

68 (1) Para un estudio del caso mencionado, véase Carmona Tinoco, Jorge Ulises, "El Caso Jorge Castañeda Gutman vs. Estados Unidos Mexicanos ante la Corte Interamericana de Derechos Humanos", Anuario Mexicano de Derecho internacional, vol. 9, 2009.

(2) El Estado de Tanzania alegó que para fortalecer la recién instaurada democracia se apostase por un sistema de partidos políticos con prohibición de las candidaturas independientes. También fue argumentado que en aras de tener en cuenta la organización territorial de Tanzania debía de existir un mínimo de miembros por cada región (Zanzíbar y Tanganica), hecho que no podría garantizarse a través de candidaturas independientes.

69 Christopher R. Mtikila v. The United Republic of Tanzania, párrafo 98.

70 Ibidem, párrafo 106.

71 En su voto particular, el magistrado Fatsah Ouguergouz critica que pareciese en el argumento de la Sala que bajo ninguna circunstancia en ningún Estado Africano se podrían prohibir las candidaturas independientes (párrafo 34, voto particular). 
cida en el artículo 10 de la Carta Africana, precisando al respecto: "It is the view of the Court that freedom of association is negated if an individual is forced to associate with others. Freedom of association is also negated if other people are forced to join up with the individual. In other words freedom of association implies freedom to associate and freedom not to associate". ${ }^{72}$

Finalmente, respecto al principio de no discriminación (artículo 2o. de la Carta Africana), elTribunal consideró que de no permitirse las candidaturas individuales se produciría un efecto discriminatorio entre los ciudadanos tanzanos en tanto solo aquellos afiliados a un partido político podrían ser electos a la Presidencia del Gobierno, al Parlamento o al Gobierno Local. Siguiendo con su razonamiento, el Tribunal determinó que la exigibilidad de tal requisito comportaría a su vez una violación del artículo 3o. de la Carta Africana: "Todos los individuos tendrán derecho a igual protección de la ley”, pues la ley ampararía un tratamiento diferente entre los ciudadanos tanzanos por razón de sus opiniones políticas.

Por todo ello, el Tribunal determinó que la República de Tanzania había incurrido en la violación de los artículos 2o., 3o., 10 y 13.1 de la Carta Africana y ordenó la inmediata adopción de las medidas constitucionales, legislativas, así como cualquier otra necesaria, para remediar las violaciones declaradas por la Sala, debiendo la Corte ser informada de las medias adoptadas al respecto.

Con respecto a las reparaciones, el Tribunal se pronunció en una resolución posterior del 13 de junio de 2014, donde la Corte, pese a reafirmar su potestad de dictar reparaciones y compensaciones en favor de las víctimas, determinó que el demante señor Mtikila no había proporcionado las pruebas oportunas sobre el lucro cesante, el coste del proceso y los daños morales alegados. Así pues, a juicio del Tribunal: "is not enough to show that a State has violated a provision of the Charter-it is also necessary to prove the damages".

Si bien, el Estado de Tanzania quedó obligado a asumir los costes de la traducción y publicación de la sentencia al inglés y al kiswahili en el Boletín Oficial del Estado y en un periódico de tirada nacional.

Según los autores consultados, esta sentencia constituye un avance significativo en el proceso de consolidación del Sistema Africano de Derechos Humanos. Así pues, en opinion de Windridge:

72 Ibidem, párrafo 113. 
Esta revista forma parte del acervo de la Biblioteca Jurídica Virtual del Instituto de Investigaciones Jurídicas de la UNAM

The Court should be commended for having delivered its first Judgment on the merits. The Judgement provides well reasoned findings for finding that the Applicants had exhausted local remedies and dismissing Tanzania's somewhat weak jurisdictional arguments. It was also encouraging to see the Court reject Tanzania's "social needs" arguments especially considering Tanzania failed to provide concrete examples of where these needs arose as discussed above. ${ }^{73}$

No obstante, se ponen de manifiesto cuestiones que han de ser perfiladas en próximos pronunciamientos. Nos referimos, entre otras, a la pauta seguida por la Corte de atribuir a cada parte del proceso la responsabilidad del pago de sus costas. Este principio puede obstaculizar la interposición de futuras demandas por individuos y ONG en tanto que sepan que, pese a vencer en el juicio, van a tener que hacer frente a las costas del proceso.

También nos referimos a la negativa del Tribunal a pronunciarse sobre la vulneración de los tratados internacionales aludidos, alegando la manifiesta violación de artículos de la Carta Africana. A este respecto, el profesor Oliviver Windridge precisa:

The Court appears to have adopted an "either/or" approach. While the Applicant's Charter rights were found to have been violated this should not mean the Applicants other rights, found under international treaties are not to be examined. There may of course be practical reasons behind the Court's decision not to go into the international treaty merits, but in deciding not to consider them in detail we are at best left wondering what the analysis may have brought up, while missing out on the possibility of further discussion and jurisprudence to guide future applications, while at worst left with the feeling the Court considers the international treaties not important. ${ }^{74}$

3. Medidas provisionales y remisión de casos desde la Comisión:

African Commission vs. Libya

En otro orden de ideas cabe destacar que la Comisión está facultada para remitir ante la Corte un caso cuando de una o más comunicaciones pre-

73 Véase Windridge, Oliver, “A Watershed Case: Mtikila and others v. Tanzania”, The ACtHPR Monitor, 2014, disponible en: http: / / www.acthprmonitor.org/a-watershed-case-mtikila-and-othersv-tanzania/ (fecha de consulta: junio de 2016).

74 Ibidem, p. 3. 
Esta revista forma parte del acervo de la Biblioteca Jurídica Virtual del Instituto de Investigaciones Jurídicas de la UNAM

sentadas ante dicha institución se deriven graves o masivas violaciones de derechos humanos. ${ }^{75}$

Dicho procedimiento fue adoptado por vez primera en el caso African Commission on Human and Peoples' Rights v. Great Socialist Peopless Libyan Arab Jamahiriya. ${ }^{76}$

Los hechos se remontan a febrero de 2011, fecha en la que las revueltas populares extendidas por todo el norte de África y Medio Oriente se transformaron en un conflicto armado en Libia. Concretamente ante la violenta represión que tuvo lugar en la ciudad de Bengasi el 16 de febrero de 2011, y que fue extendida a las principales ciudades del país, incluida la capital Trípoli, las ONG the Egyptian Initiative for Personal Rights, Human RightsWatch e INTERIGHTS presentaron un comunicado ante la comisión solicitando: ${ }^{77}$

...(i) Stop and prevent the use of unjustified lethal force against protesters, whether by the security forces, mercenaries or other bodies or individuals acting on behalf of the State; (ii) Allow people within Libya to air their grievances through peaceful protests; (iii) Allow the free flow of information, including by permitting international journalists to enter and report freely; (iv) Open up all forms of communication by restoring full use of internet, television stations, mobile phones and social networks; (v) Respect the rights of detainees; (vi) Ensure that those injured during the protests are permitted access to appropriate medical treatment; and (vii) Undertake a thorough, impartial and prompt investigation to hold accountable those responsible for these violations.

75 Así, en el Reglamento de la Comisión se establece: Artículo 84: (1) “When the Commission considers that one or more Communications relate to a series of serious or massive human rights violations, it shall bring the matter to the attention of the Assembly of Heads of State and Government of the African Union and the Peace and Security Council of the African Union. (2) The Commission may also, in conformity with Article 5 of the African Court Protocol and Rule 118 of the present Rules of Procedure, refer the matter to the African Court".

76 (1) Véase African Commission on Human and Peoples 'Rights v. Great Socialist People’s Libyan Arab Jamahiriya, Application No. 0041/2011, Order for Provisional Measures, 30 de marzo de 2011 .

(2) Otro ejemplo más reciente en el que el Tribunal Africano ha adoptado medidas provisionales es el caso African Commission on Human and Peoples' Rights v. Kenya, Application núm. $006 / 2012$.

77 Véase Sander, Barrie, “Case Summary: African Commission on Human and Peoples' Rights v. The Great Socialist People's Libyan Arab Jamahiriya”, Justice and Human Rights for Africa, 2011, disponible en: http://arcproject.co.uk/african-court-project/ (fecha de consulta: junio de 2016). 
Esta revista forma parte del acervo de la Biblioteca Jurídica Virtual del Instituto de Investigaciones Jurídicas de la UNAM

Las mismas ONG presentaron de nuevo un comunicado a la Comisión el 3 de Marzo de 2011 en el que declaraba:

Peaceful demonstrations... were violently suppressed by security forces who opened fire at random on demonstrators killing and injuring many people, that... security forces engaged in excessive use of heavy weapons and machine guns against the population, including targeted aerial bombardment and all types of attacks, and that these actions amount to serious violations of the right to life and to the integrity of persons, freedom of expression, demonstration and assembly. ${ }^{78}$

Ante estos hechos la Comisión decidió por primera vez remitir el asunto ante la Corte Africana de Derechos Humanos y de los Pueblos. ${ }^{79}$ Institución que igualmente acordó por vez primera en el ejercicio de sus facultades la adopción de medidas provisionales. Así pues, en el Caso African Commission on Human and Peoples' Rights v. Great Socialist People's Libyan Arab Jamahiriya, la Corte apoyándose en el precepto 27 del Protocolo, ${ }^{80}$ estableció por unanimidad:

1) The Great Socialist Peopless Libyan Arab Jamahiriya must immediately refrain from any action that would result in loss of life or violation of physical integrity of persons, which could be a breach of the provisions of the Charter or of other international human rights instruments to which it is a party.

2) The Great Socialist People's Libyan Arab Jamahiriya must report to the Court within a period of fifteen (15) days from the date of receipt of the Order, on the measures taken to implement this Order.

Esta actuación del Tribunal supuso un nuevo avance en la articulación del sistema africano de derechos humanos, en tanto quedó patente que pese a la ausencia de un acceso directo de individuos y ONG a la Corte, ${ }^{81}$ la Co-

78 Ibidem, p. 1.

79 Cfr. ACHRP/RES.181/2011: Resolution on the Human Rights Situation in the Great Socialist Peoples' Libyan Arab Jamahiriya

80 Cfr. artículo 27.2 del Protocolo: "In cases of extreme gravity and urgency, and when necessary to avoid irreparable harm to persons, the Court shall adopt such provisional measures as it deems necessary".

81 Recordemos que los individuos y ONG pueden presentar casos ante la Corte si el Estado contra el que se presenta el asunto ha emitido una Declaración de competencia de conformidad con el artículo 34.6 Protocolo. 
Esta revista forma parte del acervo de la Biblioteca Jurídica Virtual del Instituto de Investigaciones Jurídicas de la UNAM

misión a través de sus competencias puede salvar este escollo, remitiendo los casos presentados por individuos y ONG ante el Tribunal.

Asimismo, se hizo visible el compromiso de la Corte con la protección de los derechos humanos, al acordar la adopción de medidas provisionales sin haber sido éstas requeridas por la Comisión, en una situación que no podía esperar el lento proceso judicial, lo que comporta una legitimación de la actuación del Tribunal.

No obstante, esta sentencia también demostró las carencias del sistema. Así pues, si las resoluciones adoptadas por la Corte tienen en principio un carácter vinculante, ${ }^{82}$ la ejecución de las mismas queda en manos de la voluntad política de jefes de Estado y de gobierno de la UA. De manera que al no existir un consenso entre los jefes de Estado africanos acerca de cómo actuar respecto al régimen dictatorial de Muammar al-Qaddafi, la resolución adoptada por la Corte quedó en un brindis al sol.

A su vez, si bien la Comisión, como hemos expuesto, puede remitir casos a la Corte, también ésta se encuentra facultada para remitir casos a aquella ${ }^{83}$ Así pues, en el asunto Ekollo Moundi Alexandre vs. Republic of Cameroon and the Federal Republic of Nigeria ${ }^{84}$ ante la alegación por parte del demandante Ekollo Moundi Aiexandre domiciliado en Camerún de la violación de los artículos 3o., 5o., 6o., 7o. y 13.3 de la Carta Africana por los Estados de Camerún y Nigeria, la Corte determinó que no era competente para conocer del asunto en tanto estos Estados no habían suscrito la declaración de competencia de conformidad al 34.6 del Protocolo, acordando remitir dicho asunto a la Comisión. ${ }^{85}$

A este respecto, si para autores como Dan Juma con esta actuación se evitaría el fórum shopping del peticionario, al igual que comportaría un mecanismo a través del cual la Corte y la Comisión pudiesen colaborar mu-

82 Hay que mencionar al respecto que sigue generando dudas si las medidas provisionales adoptadas por el Tribunal carecerían de un carácter obligatorio, pues ni en el Protocolo ni en el Reglamento de la Corte se le atribuyen expresamente tal carácter.

83 Nótese que en el artículo 6o. del Protocolo, bajo la rúbrica Admisibilidad de los casos, se establece: (3) "The Court may consider cases or transfer them to the Commission".

84 Véase Application 008/2011 Ekollo Moundi Alexandre v Republic of Cameroon and the Federal Republic of Nigeria

85 Cabe mencionar que en aplicación del artículo 22 del Protocolo, en el que se expresa «If the judge is a national of any State which is a party to a case submitted to the Court, that judge shall not hear the case», se acordó la recusación del juez Elsie N. Thompson de nacionalidad nigeriana. 
Esta revista forma parte del acervo de la Biblioteca Jurídica Virtual del Instituto de Investigaciones Jurídicas de la UNAM

tuamente en aras de establecer una administración de justicia eficaz, ${ }^{86}$ para autores como Fatsah Ouguergouz:

In deciding not to rule on the merits of a case over which it has jurisdiction, the African Court could however be opening the door to a veritable deniable of justice; the referral of the case to the Commission for determination on the merits would not suffice to forestall such a denial of justice since only the Court does have powers of a judicial nature. ${ }^{87}$

4. Opiniones consultivas: The African Committee of Experts on the Rights andWelfare of the Child, Coalition on International Criminal Court LTD / GTE

En virtud del artículo 4o. del Protocolo, entre sus funciones, el Tribunal Africano no sólo tiene encomendada una competencia contenciosa, sino también consultiva. ${ }^{88}$ Así pues, hasta mayo de 2016 la Corte ha emitido su parecer sobre siete casos. ${ }^{89}$

A este respecto, cabe destacar la consulta núm. 002/2013 The African Committee of Experts on the Rights andWelfare of the Child before the African Court of Human and Peoples> Rights ${ }^{90}$ en la que el Comité Africano de Expertos sobre los Derechos y el Bienestar del Niño ${ }^{91}$ solicita el parecer de la Cor-

86 Véase Dan, Juma, "Provisional Measures Under the African Human Rights System: The African Court's Order Against Libya”, Wisconsin International Law Journal, vol. 30, 2012, p. 354.

87 Cfr. opinión particular del juez Fatsah Ouguergouz, Application 008/2011 Ekollo Moundi Alexandre v Republic of Cameroon and the Federal Republic of Nigeria (Párr. 34).

88 Nótese que en el artículo 4o. del Protocolo se establece: "At the request of a Member State of the $O A U$, the $O A U$, any of its organs, or any African organization recognized by the $O A U$, the Court may provide an opinion on any legal matter relating to the Charter or any other relevant human rights instruments, provided that the subject matter of the opinion is not related to a matter being examined by the Commission".

89 El parecer del Tribunal sobre los casos presentados con base en su jurisdicción consultiva pueden ser objeto de consulta en: http: / / en.african-court.org/\#advisory-opinions (fecha de consulta: mayo de 2016).

90 Véase Request No 002/2013 - The African Committee of Experts on the Rights and Welfare of the Child on the Standing of the African Committee of Experts on the Rights and Welfare of the Child before the African Court of Human and Peoples' Rights.

91 Dicho órgano es creado en virtud del mandato contenido en los artículos 32 a 36 de la Carta Africana de los Derechos y Bienestar del Niño. A este respecto, el artículo 32 ordena la creación de "un Comité Africano de Expertos sobre los Derechos y el Bienestar del Niño 
Esta revista forma parte del acervo de la Biblioteca Jurídica Virtual del Instituto de Investigaciones Jurídicas de la UNAM

te sobre su legitimación activa en relación con la jurisdicción consultiva y contenciosa de la Corte.

Con respecto a la competencia consultiva, el Tribunal argumenta que en virtud del artículo 4o. del Protocolo, los Estados miembros de la Unión Africana (UA), la propia UA, algunos de sus órganos o cualquier otra organización africana reconocida por la UA puede requerir una opinión consultiva ante la Corte. Por ende, en tanto el Comité Africano de Expertos sobre los Derechos y el Bienestar del Niño es uno de los órganos de la Unión Africana, tiene reconocida la legitimación activa en materia consultiva. ${ }^{92}$

Con respecto a la jurisdicción contenciosa, el Tribunal apunta al artículo 5o. del Protocolo para determinar que el Comité no se encuentra entre los sujetos facultados para remitir a la Corte un caso contencioso. ${ }^{93}$

El Tribunal arguye que, si bien no encuentra razón para que dicho órgano no pueda presentar tales casos, en tanto que dentro de la ratio materiae del Tribunal se encuentra la Carta Africana de los Derechos y Bienestar del Niño, al mismo tiempo que el mandato que se le encomienda es reforzar y fortalecer la actuación de la Comisión y por extensión el marco africano de derechos humanos, en el que quedaría incluido el Comité, el Tribunal razona que "where a treaty sets out an exhaustive list, this cannot be interpreted to include an entity that is not listed, even if it has the same attributtes. Therefore, the Court cannot substitute itself and assume the functions of the legislatura" ${ }^{94}$

No obstante, el Tribunal en su dictamen señala como "highly desirable" que el Comité sea facultado para presentar casos contenciosos ante la Corte, actuación que robustecería su cometido. ${ }^{95}$

dentro del marco de la Organización para la Unidad Africana, para promover y proteger los derechos y el bienestar del niño”.

92 Ibidem, párrafos 45 y ss.

93 Atendiendo al artículo 5o. del Protocolo, tienen legitimación activa ante la Corte en materia contenciosa: "a)The Commission b)The State Party which had lodged a complaint to the Commission c) The State Party against which the complaint has been lodged at the Commission d)The State Party whose citizen is a victim of human rights violation e) African Intergovernmental Organizations".

94 Ibidem, párrafos 98 y 99.

95 Cabe mencionar que en el Protocolo del TAJDH sí se permite expresamente que el Comité pueda presentar casos ante la Corte (artículos 29 y 30 del Protocolo delTAJDH). 
Esta revista forma parte del acervo de la Biblioteca Jurídica Virtual del Instituto de Investigaciones Jurídicas de la UNAM

\section{LA RELACIÓN ENTRE ELTRIBUNAL AFRICANO Y LA COMISIÓN}

Si bien tras los primeros años de la creación de la Corte, autores como Udombana, Sánchez de Muniáin y Mbondenyi ${ }^{96}$ apreciaron que el vínculo existente entre estas dos instituciones necesitaba de una clarificación, pues en gran medida tenían encomendadas funciones redundantes sin llegar a establecerse un auténtico mecanismo de asistencia y cooperación, con la aprobación del Reglamento de la Comisión ${ }^{97}$ y el Reglamento de Funcionamiento interno de la Corte ${ }^{98}$ se van a solucionar en gran medida las incertidumbres planteadas.

El principio general que guía las relaciones entre la Corte y la Comisión se encuentra recogido en el artículo segundo del Protocolo del Tribunal, en el que se establece: "The Court shall, bearing in mind the provisions of this Protocol, complement the protective mandate of the African Commission on Human and Peoples' Rights, conferred upon it by the African Charter on Human and Peoples' Rights, hereinafter referred to as "the Charter"”.

Asimismo, en el Reglamento de la Comisión se detalla tres situaciones en virtud de las cuales la Comisión puede remitir a la Corte los casos sometidos a su jurisdicción:

- Si la Comisión ha dictado su parecer en relación con un comunicado remitido por un Estado miembro de la UA, una ONG o un individuo y considera que el Estado acusado no ha cumplido o es reacio a cumplir con las recomendaciones establecidas por la Comisión, dicho órgano puede remitir el caso ante el Tribunal Africano e informar a las partes en el proceso. ${ }^{99}$

De esta forma, en tanto que las comunicaciones de la Comisión tienen un carácter recomendatorio, se persigue que la Corte dicte una sentencia vinculante, teniendo el Estado la obligación de proceder a su cumplimento. No obstante, en opinión del autor Dan Juma:

96 Véase Mbondenyi, op. cit., p. 387.

97 Nótese que se dedica la Parte IV del Reglamento de la Comisión a perfilar las relaciones entre el Tribunal y la Comisión, concretamente en los artículos 114 a 123.

98 En el Reglamento de Funcionamiento Interno de la Corte, las relaciones entre el Tribunal y la Comisión quedan establecidas en el artículo 29.

99 Artículo 118.1 Reglamento de la Comisión. 
The pitfall of this procedure is that it is essentially a "follow-up" or an "implementation" mechanism, coming late in the life of a case. The second problem is the assumption that referral to the African Court-where the African Commission has not succeeded-would ensure compliance. Indeed, complex factors are often at play in human rights regimes, and the juridical nature of the decisions per se should not be deemed as the key driver toward compliance. $^{100}$

- Si la Comisión ha acordado adoptar medidas provisionales en aras de prevenir daños irreparables a la víctima de la violación alegada y éste órgano considera que el Estado no ha cumplido con las medidas provisionales requeridas, la Comisión podrá poner en conocimiento de la Corte el caso en cuestión. ${ }^{101}$

Siguiendo con la opinión del citado autor "the caveats lodged above in relation to the first category of cases, and the history of noncompliance with the African Commission's interim orders raises doubts whether this second layer of legality, or the bolstering of such orders' juridical status through the African Court will induce better compliance". ${ }^{102}$

- De presentarse ante la Comisión una comunicación que revele la existencia de graves o masivas violaciones de derechos humanos, la Comisión podrá remitir el caso ante el Tribunal Africano. ${ }^{103}$ Este es el procedimiento seguido en el asunto African Commission on Human and Peoples ‘ Rights v. Great Socialist People’s Libyan Arab Jamahiriya, analizado en el epígrafe anterior.

Nótese que la Carta Africana, en casos de graves o masivas violaciones de derechos humanos, prevé que la Comisión debe en primer lugar comunicar la situación a la Asamblea de Jefes de Estado y de Gobierno, quienes si lo consideran oportuno pueden encomendar a la Comisión la realización de un estudio a fondo sobre esos casos (artículo 58 Carta Africana). Si bien la Comisión ha hecho uso de este precepto, la respuesta de la Asamblea ha sido siempre la indiferencia. Así pues, el mecanismo previsto en el Reglamento de la Comisión, permitiendo a dicho organismo remitir tales casos

100 Véase Dan Juma, op. cit., p. 351.

101 Artículo 118.2 del Reglamento de la Comisión.

102 Ibidem, p.352.

103 Artículo 118.3 del Reglamento de la Comisión. 
Esta revista forma parte del acervo de la Biblioteca Jurídica Virtual del Instituto de Investigaciones Jurídicas de la UNAM

ante la Corte, ha supuesto un avance en el proceso de protección de los derechos humanos en África.

Otro rasgo distintivo es la potestad atribuida al Tribunal de presentar ante la Comisión, si así lo considerara oportuno, en cualquier momento del procedimiento, los casos sometidos a su jurisdicción. ${ }^{104}$ Con esta previsión, en opinión de los autores consultados, se evitaría el fórum shopping por el peticionario.

Asimismo, se potencia la complementariedad entre la Comisión y el Tribunal posibilitando que con anterioridad a la admisibilidad de los casos sometidos por individuos y ONG ante la Corte, ésta pueda solicitar el parecer de la Comisión. En esta línea, para evitar un pronunciamiento contradictorio, en tanto la Comisión también tiene atribuida competencia contenciosa, consultiva y arbitral, se prevé que dicho organismo no pueda considerar ninguna comunicación que se encuentre aún pendiente ante la Corte, a menos que la comunicación haya sido formalmente retirada. ${ }^{105}$ Además, siempre que se requiera a la Comisión interpretar las disposiciones de la Carta Africana, ésta deberá inmediatamente informar al presidente del Tribunal. ${ }^{106}$

Así pues, con estas disposiciones se ha logrado completar y reforzar mutuamente las funciones encomendadas a la Comisión y a la Corte, al mismo tiempo que se posibilita el conocimiento por parte del Tribunal de los casos presentados por ONG e individuos, quienes en principio no tienen acceso directo a la Corte. ${ }^{107}$ Atendiendo a las palabras del profesor Nsongura J. Ubunda, con base en estos preceptos, "the Commission is the preliminary body for the settlement of disputes between two states or between an individual and a state.

104 Artículo 6.3 del Protocolo.

105 Artículos 6.1 Protocolo y 123 de la Comisión. Cabe mencionar que en aras de asegurar la cooperación entre ambas instituciones en el artículo 33 del Protocolo se ordena consultar a la Comisión al redactar el Reglamento de la Corte: "The Court shall draw up its Rules and determine its own procedures. The Court shall consult the Commission as appropriate".

106 Artículo 116 del Reglamento de la Comisión. En relación con la competencia consultiva, se plantea la duda de si la Comisión tienen legitimación activa para solicitar ante el Tribunal opiniones consultivas. Para autores como Khulekani y D. Juma al ser un órgano de la UA sí tendría competencia. En cambio, autores como Saavedra Álvarez sostienen la postura contraria, en tanto que en el Protocolo no se menciona entre los legitimarios activos a la Comisión. Véase artículo 4o. del Protocolo.

107 Véase Moyo, Khulekani, "Justice for All: Realising the Promise of the Protocol Establishing the African Court on Human and Peoples' Rights”, SALC Handbook Series, 2014, pp. 9 y ss. 
Esta revista forma parte del acervo de la Biblioteca Jurídica Virtual del Instituto de Investigaciones Jurídicas de la UNAM

In other words, the Commission serves as an organ of investigation to help the Court's judgment on the case. It will play a prominent role as a filter mechanism". ${ }^{108}$

No obstante, aún persisten cuestiones no resueltas, entre las que destacan la posibilidad o no de que el demandante pueda acudir a la Comisión una vez que la Corte haya fallado a favor del demandado.

\section{CONCLUSIÓN Y ESTADO DE LA CUESTIÓN}

Con el establecimiento del Tribunal Africano de Derechos Humanos y de los Pueblos se dio un paso transcendental en la consolidación del sistema africano de derechos humanos, en tanto se puso de manifiesto que los gobiernos africanos "empezaban a tomar los derechos humanos en serio", ${ }^{109}$ ejecutando de una vez por todas la idea ya planteada en la Conferencia de Lagos de 1961 y desechada bajo el pretexto de poner en peligro el principio de soberanía estatal.

Con la creación de esta institución jurisdiccional se arroja esperanza en el continente africano para la protección universal de los derechos humanos. Así pues, por primera vez se crea un órgano judicial que emite decisiones obligatorias, con una actuación transparente y pública. Esta última característica constituye una diferencia sustancial respecto a la Comisión, puesto que según el artículo 59 de la Carta Africana sus actuaciones son confidenciales hasta que la Asamblea de Jefes de Estado y de Gobierno decida lo contrario. De esta forma, a través de la denuncia pública, se articula un medio eficaz para hacer presión sobre los Estados en el cumplimiento de sus sentencias. ${ }^{110}$

108 Véase Udombanat, Nsongurua J., Toward, cit., p. 98.

109 Esta frase fue utilizada por primera vez por el profesor Udombana al adoptarse la Carta Africana: "The African Charter was the first serious and potentially significant attempt by African leaders towards 'taking rights seriously", véase Udombana, Nsongurua, "Can the Leopard Change Its Spots? The African Union Treaty and Human Rights", University, American International Law Review,Washington, vol. 17, núm. 6, 2002, pp. 1206 y 1207.

110 Véase Yerima, Timothy F., "New Trends In The African Human Rights System: Prospects Of African Regional Human Rights Courts”, Global Journal of Human Social Science Arts \& Humanities, vol. 12, núm. 2, 2012, p. 75. 
Asimismo, como establece V. Frans, para la protección de los derechos humanos en cualquier región se requiere una jurisprudencia regional de derechos humanos. Más si se tiene en cuenta la formulación restrictiva de numerosos derechos en la Carta Africana. ${ }^{111}$

En este orden de ideas, el desarrollo y consolidación de su jurisprudencia, facilitará e impulsará al mismo tiempo la labor llevada a cabo por los tribunales internos de los Estados africanos en la protección de los derechos humanos, así como la actuación de los tribunales de comunidades subregionales, que como ECOWAS, tienen entre sus competencias la Carta Africana de Derechos Humanos y de los Pueblos.

No obstante, pese a estos avances, en opinión de autores como Ogwezzy Michael: "The challenges facing the merged courts of Justice and Human rights are numerous and breathtaking". ${ }^{112}$

- En primer lugar, debemos destacar que la efectividad del sistema dependerá en gran medida de la voluntad política de los gobiernos africanos para acatar y cumplir las sentencias dictadas por la Corte. Si bien, las decisiones emanadas del TADHP son obligatorias y se ha logrado establecer un mecanismo represivo a través del cual el Consejo Ejecutivo de la UA es el órgano encargado de hacer cumplir las resoluciones, en última instancia dicho organismo actúa en nombre de la Asamblea de Jefes de Estado y de Gobierno de la UA, por lo que dependerá de la voluntad política de esta institución hacer efectivos los pronunciamientos de la Corte.

A ello hay que añadirle el caos institucional existente. Se intentará explicar de forma resumida. Junto al Tribunal Africano de Derechos Humanos y de los Pueblos se aprobó en 2003 el Protocolo por el que se crea un Tribunal de Justicia de la Unión Africana entrando en vigor en 2009. No obstante, dicho Tribunal no se encuentra actualmente operativo y probablemente nunca lo esté, pues los jefes de Estado y de gobierno de la UA acordaron en 2004 la fusión de este Tribunal con el Tribunal Africano de Derechos Humanos y de los Pueblos dando lugar

111 Véase Frans, V., "The Relevance of the Inter-American Human Rights System for Africa”, Afric. Journal of Inter'l \& Comp. Law, 1999, p.660.

112 Véase Ogwezzy, Michael, "Challenges and Prospects of the African Court of Justice and Human Rights”, Jimma University Journal of Law, vol. 6, 2014, p. 19. 
al Tribunal Africano de Justicia y Derechos Humanos. El Protocolo por el que se crea este Tribunal refundido, el Protocolo del Estatuto de la Corte Africana de Justicia y Derechos Humanos, requiere para su entrada en vigor 15 ratificaciones, y actualmente sólo ha sido ratificado por 5 Estados.

El caos institucional no se queda ahí. En 2014 tuvo lugar la aprobación de un nuevo Protocolo (Protocolo de Reforma del Protocolo del Estatuto de la Corte Africana de Justicia y Derechos Humanos o Protocolo de Malabo) por el que se le atribuye competencia penal al Tribunal refundido y se modifica el nombre del mismo, pasándose a denominar Tribunal Africano de Justicia y Derechos Humanos y de los Pueblos (TAJDHP). Para su entrada en vigor se requieren 15 ratificaciones y en la fecha de redacción de este artículo ningún Estado había dado el primer paso. Dicho Protocolo ha generado acervadas críticas por una parte de la comunidad científica en tanto que otorga inmunidad a los jefes de Estado y de gobierno y mantiene un inaudito mutismo en su relación con la Corte Penal Internacional (CPI), más teniendo en cuenta que 33 Estados africanos miembros de la UA son a su vez Estados parte en la CPI.

No obstante, como hemos sido testigos en estas líneas, en la Unión Africana la creación de instituciones, la atribución de competencias y los procesos de reforma no se caracterizan por su celeridad, por lo que hay tiempo para replantear la relación con la CPI y no tirar por la borda todos los progresos que en el ámbito de los derechos humanos se han llevado a cabo. Así pues, debemos insistir que actualmente sólo se encuentra en vigor el Tribunal Africano de Derechos Humanos y de los Pueblos (TADHP) y esperamos que durante los previsibles siguientes años los puntos más controvertidos del Protocolo de Malabo sean modificados o modulados.

- El segundo pilar para la efectividad de las instituciones jurisdiccionales africanas es la independencia, imparcialidad y competencia de sus magistrados. Si bien, en el Protocolo del TADHP se establecen una serie de preceptos que velan por la objetividad del juez, habrá que comprobar, en la práctica, si los magistrados no atienden a las pretensiones de los Estados que los designan, como ocurrió en un principio con la Comisión. 
También cabe mencionar que si consideramos el número potencial de casos que pueden llegar a ser presentados ante elTAJDHP y las violaciones de derechos humanos que actualmente se están produciendo en África y que podrían quedar bajo jurisdicción del Tribunal, el hecho de que en el Protocolo de Malabo haya previsto que el mandato de los jueces sea a tiempo parcial ha sido objeto de acervadas críticas por los autores. ${ }^{113}$

- Asimismo, debido a la extensión del continente africano y al analfabetismo de una gran parte de la población, las instituciones creadas por la Unión Africana son desconocidas para la mayoría de las víctimas de violaciones de derechos humanos. En este sentido, la Comisión y la Corte, ayudándose del apoyo de organizaciones no gubernamentales, deben llevar a cabo una tarea de promoción de sus actividades y de puesta en valor de los beneficios que para el pueblo africano conlleva la creación de tales instituciones, evitando exclusivamente centrarse en el ámbito universitario y extendido su actuación hacia las zonas rurales. ${ }^{114}$

- Otro de los grandes retos es la adecuada financiación de las instituciones africanas. Si ya la Comisión durante sus más de veinticinco años de existencia ha expresado la insuficiencia de fondos para hacer frente a las tareas encomendadas, en el momento actual, donde se pretende poner en funcionamiento un Tribunal compuesto por tres secciones (de Justicia, Derechos Humanos y Penal), se requiere más que nunca que los Estados africanos cumplan sus compromisos financieros para evitar que las funciones atribuidas no puedan ser efectuadas por falta de fondos. ${ }^{115}$

- En otro orden de ideas, el TADHP debe seguir los pasos de la Comisión, y apostar por una actuación innovadora que permita suplir las

113 No obstante, hay que tener presente que en el artículo 9o. del Protocolo del TAJDHP se encomienda al presidente y al vicepresidente del Tribunal el desempeño de sus funciones a tiempo completo.

114 Véase Heyns, Christof, "The African Regional Human Rights Sistem: In Need of Reform?”, African Human Rights Law Journal, vol. 2, 2001, p. 172.

115 Cabe mencionar que instituciones como la Unión Europea, la Agencia de Desarrollo Internacional de Canadá (CIDA), la Agencia Alemana de Cooperación Técnica (GTZ), el Instituto Danés de Derechos Humanos y Open Society, entre otras, han estado colaborando económicamente con la UA para promover la labor de sus instituciones. 
carencias de los preceptos de la Carta de Banjul y de su Protocolo. Así pues, ya en la primera sentencia dictada por el TADHP, el magistrado Fatsah Ouguerouz adoptó una actitud reformadora estableciendo que, frente a la necesidad de que los Estados emitan expresamente una declaración de competencias para que los individuos y ONG pudieran presentar casos ante la Corte, se podría entender que el Protocolo permite la aplicación del principio fórum prorrugatum. En esta línea, el TADHP debería hacer frente a lagunas como la obligatoriedad o no de las medidas provisionales adoptada por el Tribunal, siguiendo el camino establecido por la Corte Europea de Derechos Humanos en el Caso Mamatkulov and Abdurasulovic v. Turkey: "A failure to comply with interim measures had to be regarded as preventing the Court from effectively examining the applicant's complaint and as hindering the effective exercise of his or her right and, accordingly, as a violation of Article 34". ${ }^{116}$

- Igualmente, la UA y los Estados africanos han de asumir un compromiso real de potenciar el progreso económico y las condiciones sociopolíticas en África como prerrequisito para conseguir un adecuado respeto por los derechos humanos. Es una quimera pretender instaurar súbitamente estándares occidentales de derechos humanos en una región asolada por golpes de estado, hambre, corrupción, desequilibrios económicos, terrorismo y extremismos religiosos.

- Por último, debemos tener en cuenta que mientras la sede de la Comisión ha sido fijada en Banjul (Gambia), la sede del TADHP se ha establecido temporalmente en Arusha (Tanzania), existiendo más de 8000 kilómetros de distancia entre ambas. Así pues, en opinión de autores como Esther Kamau "the African system has a lot to learn from the Inter-American system in the sense that the distance between the seat of the Inter-American Court and the Inter-American Commission in San Jose, Costa Rica, and Washington D. C respectively has been attributed for the «initial lack of cooperation» between the two organs". ${ }^{117}$

116 Véase Mamatkulov and Abdurasulovic v. Turkey, Application Nos. 46827/99 y 46951/99, párr. 110.

117 Véase Kamau, Esther, "Individual Complaint Mechanism in the Jurisprudence of the African Human Rights System Vis a Vis the European and the Inter-American Human Rights Systems: Inadequacies and Prospects”, International Justice Resource Center, 2011, p. 93. 
De tal manera, que sólo si el Tribunal Africano sigue apostando por la defensa de los derechos humanos sin vacilación y logra superar los retos que hemos tenido la ocasión de exponer, podrá consolidarse el sendero para hacer realidad la frase tantas veces repetida por Nelson Mandela: "Sueño un África en paz consigo misma”.

\section{BiBLIOGRAFÍA}

DAN, Juma, "Provisional Measures Under the African Human Rights System: The African Court's Order Against Libya”, Wisconsin International Law Journal, vol. 30, 2012.

Heyns, Christof, "The African Regional Human Rights System: In Need of Reform?”, African Human Rights Law Journal, vol. 1, núm. 2, 2001.

Jalloh, Charles, "International Decision: Michelot Yogogombaye v. The Republic of Senegal”, American Journal of International Law, vol. 104, núm. 4, 2010.

KebA, M'Baye, "Les Droits de L'homme En Afrique”, Revue Internationale de Droit Comparé, vol. 45, núm. 3, 1993.

KAMAU, Esther, "Individual Complaint Mechanism in the Jurisprudence of the African Human Rights System Vis a Vis the European and the InterAmerican Human Rights Systems: Inadequacies and Prospects", International Justice Resource Center, 2011.

MbOndenYI, Morris Kiwinda, "Investigating the Challenges in Enforcing International Human Rights Law in Africa: Towards an Effective Regional System”, University of South Africa, Pretoria, 2006.

Moyo, Khulekani, "Justice for All: Realising the Promise of the Protocol Establishing the African Court on Human and Peoples' Rights", SALC Handbook Series, 2014.

Naldi G. y Magliveras K., "Reinforcing the African System of Human Rights: The Protocol on the Establishment of a Regional Court of $\mathrm{Hu}$ man and Peoples' Rights”, Netherlands Quarterly of Human Rights, 1998. 
OGWEZZY, Michael, "Challenges and Prospects of the African Court of Justice and Human Rights”, Jimma University Journal of Law, vol. 6, 2014.

SaAVEDra Álvarez, Yuria, "El sistema africano de derechos humanos y de los pueblos. Prolegómenos", Anuario Mexicano de Derecho Internacional, vol. 8, 2008.

SAn Martín SANChez de Munián, Laura, "Comentarios acerca de la creación de un Tribunal Africano de Derechos Humanos y de los Pueblos”, Anuario de Derecho Internacional, vol. 15, 1999.

SANDER, Barrie, "Case Summary: African Commission on Human and Peoples' Rights v. The Great Socialist People's Libyan Arab Jamahiriya”, Justice and Human Rights for Africa, 2011.

Udombana, Nsongurua, "Toward the African Court on Human and People' Rights: Better Late than Never", Yale Human Rights and Development Journal, vol. 3, 2014.

-, "Can the Leopard Change Its Spots? The African Union Treaty and Human Rights", University, American International Law Review, Washington vol. 17, núm. 6, 2002.

Windridge, Oliver, "A Watershed Moment for African Human Rights: Mtikila \& Others v Tanzania at the African Court on Human and Peoples' Rights", Oxford Human Rights Hub, 2015.

Wright, Rebecca, "Finding an Impetus for Institutional Change at the African Court on Human and Peoples' Rights", Berkeley Journal of International Law, vol. 24, 2006.

Yerima, Timothy F., "New Trends In The African Human Rights System: Prospects Of African Regional Human Rights Courts", Global Journal of Human Social Science Arts \& Humanities vol. 12, núm. 2, 2012. 